\title{
Guidance of Neuronal Growth Cones in the Grasshopper Embryo. II. Recognition of a Specific Axonal Pathway by the aCC Neuron
}

\author{
Sascha du Lac, ${ }^{1}$ Michael J. Bastiani, and Corey S. Goodman \\ Department of Biological Sciences, Stanford University, Stanford, California 94305
}

In the previous paper, we experimentally analyzed the selective affinity of the pCC growth cone for the MP1/dMP2 fascicle in the grasshopper embryo. Here we describe a similar experimental analysis demonstrating the selective affinity of the aCC growth cone for the $U$ fascicle. These results support the labeled-pathways hypothesis.

The 2 sets of cell ablation experiments argue against the simple location of axons, the simple timing of axon outgrowth, and/ or simple quantitative differences in the expression of a common surface label being the major determinant in pathway selection by neuronal growth cones. Rather, these 2 examples of growthcone specificity, together with previous studies on the $\mathbf{G}$ growth cone, suggest that neuronal recognition molecules are differentially expressed on the surfaces of different embryonic axonal pathways and tracts and are used to guide growth cones through a series of choice points to their targets.

Previous studies of the grasshopper embryo revealed that neuronal growth cones make specific choices as they turn and extend along particular axonal pathways towards their targets; these specific and often divergent pathway choices are among the earliest recognition events during neuronal development (Raper et al., 1983a, b). The results suggested that the selective affinities of filopodia guide growth cones, and that these differential affinities give rise to the stereotyped patterns of selective fasciculation (Bastiani et al., 1984). From these ideas emerged the labeled-pathways hypothesis, which predicts that neighboring axon fascicles in the embryonic neuropil are differentially labeled by surface recognition molecules used by growth cones for their selective fasciculation (Goodman et al., 1982; Raper et al., $1983 \mathrm{~b}$ ). This hypothesis was experimentally tested and confirmed for the $G$ growth cone (Raper et al., 1984).

In the previous paper we began by describing the development of the first 3 longitudinal axon fascicles and the intersegmental nerve in the grasshopper embryo. These axonal pathways initially contain the axons of 7 identified neurons. The growth cones of these neurons are able to distinguish one another's surfaces and, by their specific interactions, selectively fasciculate with one another to form these 3 axon bundles. We next described and experimentally tested the selective affinity of the growth cone of one of these neurons, the $\mathrm{pCC}$, for the initial 2 axons (MP1 and dMP2) in 1 of these first 3 axon bundles (the MP1/dMP2 fascicle).

\footnotetext{
Received Feb. 18, 1986; revised Apr. 25, 1986; accepted May 5, 1986.

We thank Frances Thomas for technical assistance. This research was supported by an NIMH Traineeship (S.d.L.), Scholars Award from the F.E.S.N. Foundation (M.J.B.), and grants and awards from the NIH, NSF, March of Dimes, and the McKnight Foundation (C.S.G.)

Correspondence should be addressed to Corey $S$. Goodman at the above address.

1 Present address: Department of Neurobiology, Stanford University School of Medicine, Stanford, CA 94305.
}

Copyright (C) 1986 Society for Neuroscience $0270-6474 / 86 / 123532-10 \$ 02.00 / 0$
While the $\mathrm{pCC}$ growth cone extends anteriorly along the MP1/ dMP2 fascicle, the growth cone of its sibling, the aCC neuron, behaves quite differently when faced with the same environment. In this paper, we focus on the pathway choice of the $\mathrm{aCC}$ growth cone, examining its filopodial extension and contacts and experimentally testing its specificity. We end by discussing the applicability of our model, derived from a reductionist approach using a simple developing nervous system, to the development of more complex nervous systems. Some of these results have been briefly reviewed previously (Bastiani et al., 1985; Goodman et al., 1984).

\section{Materials and Methods}

All methods used here are described in the previous paper.

\section{Results}

\section{Selective affinity of the aCC growth cone for the U fascicle}

As described in the previous paper, the first 3 longitudinal axon fascicles in the grasshopper embryo are the vMP2 fascicle, the MP1/dMP2 fascicle, and the $U$ fascicle. The growth cones of the sibling aCC and pCC neurons make divergent choices when confronted with these first 3 axon bundles. The pCC growth cone extends anteriorly along the MP1 and dMP2 axons in the MP1/dMP2 fascicle while the aCC growth cone extends posteriorly along the $\mathrm{U} 1$ and $\mathrm{U} 2$ axons in the $\mathrm{U}$ fascicle.

By $31 \%$ of embryonic development (see Fig. 3 in the previous paper), the growth cones of the sibling aCC and pCC neurons make divergent choices, ultimately fasciculating with different axon bundles. The pCC growth cone extends anteriorly as it fasciculates with the MP1 and dMP2 axons. In contrast, the growth cone of the aCC neuron remains relatively stationary during this period $(30-32 \%)$ as it points anteriorly. However, after 10-15 hr (by 33\%), the behavior of the aCC growth cone dramatically changes. This change is precisely correlated with the appearance of the U1 and U2 growth cones on the dorsal surface within $10 \mu \mathrm{m}$, and thus within filopodial reach, of the aCC growth cone.

The cell bodies of the U1 and $\mathrm{U} 2$ neurons are deep in the ventral cell body layer, far away from the dorsal filopodia of the aCC neuron. The U1 and U2 neurons extend growth cones dorsally that arrive at the dorsal basement membrane just lateral to the MP1/dMP2 fascicle. They do not fasciculate with the 2 existing longitudinal axon fascicles (vMP2 and MP1/dMP2) but rather turn posteriorly and pioneer a third axon fascicle (the $U$ fascicle) just a few microns lateral to the MP1/dMP2 fascicle (33-35\%).

Once the $\mathrm{U}$ growth cones reach the basement membrane and begin extending posteriorly, the aCC growth cone begins to change its direction, extending laterally towards them and crossing over to the MP1/dMP2 fascicle. The aCC growth cone continues to extend posteriorly along the $U$ axons. By $35 \%$, the $U$ axons have turned laterally, pioneering the intersegmental nerve. The aCC 
growth cone turns laterally along the same pathway (see the following paper).

To confirm and further extend our light-level observations of the behavior of the aCC growth cone, we examined electron micrographs taken from serial sections of these neurons and fascicles at $32-35 \%$ of development. In the example shown in Figures 1 and 2, the aCC and MP1 neurons (and a glial cell) were filled with HRP in a $35 \%$ embryo. This embryo was fixed and embedded in plastic for the EM. However, before the tissue was sectioned, the whole-mount embryo was photographed in plastic from the dorsal surface (Fig. 1). Serial thin sections were then cut from the level of the MP1 cell body (Fig. $2 A$ ) to the level of the next posterior segment border. The electron micrographs shown in Figure 2 were taken at the levels indicated by the arrows and letters B, C, and D in Figure 1. The growth cone of the darkly filled aCC neuron has reached the segment border, where it is about to turn laterally. The lightly filled MP1 axon lies medial to the aCC axon. A connective glial cell (gl) is also darkly filled (see the following paper).

In Figure $2 D$ (level D in Fig. 1), 3 longitudinal axon fascicles are seen in the connective: the vMP2 fascicle, containing the single vMP2 axon; the MP1/dMP2 fascicle, containing only the MP1 and dMP2 axons at this level (the pCC axon is more anterior); and the $U$ fascicle, containing the $2 \mathrm{U}$ axons and the aCC axon. As we examine sections more anterior in the ganglionic neuropil (Fig. $2 A$ ), we see, in contrast to the simplicity of the longitudinal connective, more growth cones and axons extending in a variety of directions, many of which form both commissural fascicles and additional longitudinal fascicles.

In a section taken anterior to the aCC, at the level of the MP1 cell body (Fig. $2 A$ ), the pCC axon has joined the MP1/dMP2 fascicle. Ventromedial to this fascicle lies the vMP2 fascicle, which contains the axons of the VMP2 neurons from this and the next posterior segment. In more posterior sections of the connective (Fig. 2, $B-D$ ), the MP1/dMP2 and vMP2 fascicles maintain their positions relative to each other. At these levels, the vMP2 fascicle contains only the vMP2 axon from the next posterior segment. In the section shown in Figure $2 B$, the Q1 axon has contacted the MP1/dMP2 fascicle as it extends towards the midline.

The photomicrographs confirm that the aCC fasciculates with the $U$ axons (Fig. $2 D$ ). As the aCC axon leaves the cell body (Fig. 2B), it extends laterally along the basement membrane, passing over the vMP2 and MP1/dMP2 fascicles. The aCC axon adheres to the basement membrane (Fig. $2 C$ ) until it reaches the $\mathrm{U}$ fascicle. At this point, the axon fasciculates with the $\mathrm{U} 1$ and $\mathrm{U} 2$ axons (Fig. 2D).

\section{Filopodial extension and contacts by the aCC growth cone}

We observed the filopodia of the aCC growth cone at the light level by fixing the embryo, injecting Lucifer yellow (LY) into the aCC cell body, fixing the embryo again, incubating the embryo in the anti-LY antibody (Taghert et al., 1982), and processing the embryo for HRP immunocytochemistry. Two examples of this method are shown in Figure 3. This method has several advantages over conventional LY injections. First, impalements of embryonic neurons, particularly those with growth cones at or near the cell body, often cause filopodia to retract; this problem is eliminated by first fixing the embryo before impalement (Thomas et al., 1984). Second, the LY halos often obscure the full extent of the filopodia; this problem is eliminated by using the anti-LY antibody and HRP immunocytochemistry (Taghert et al., 1982).

The shape of the aCC growth cone and the extent of its filopodia change dramatically between 32 and $35 \%$ of development (Fig. 4). At 32\%, numerous filopodia radiate anteriorly and laterally from the aCC cell body. At the light- and electronmicroscopic levels, these filopodia extend in a planar array,

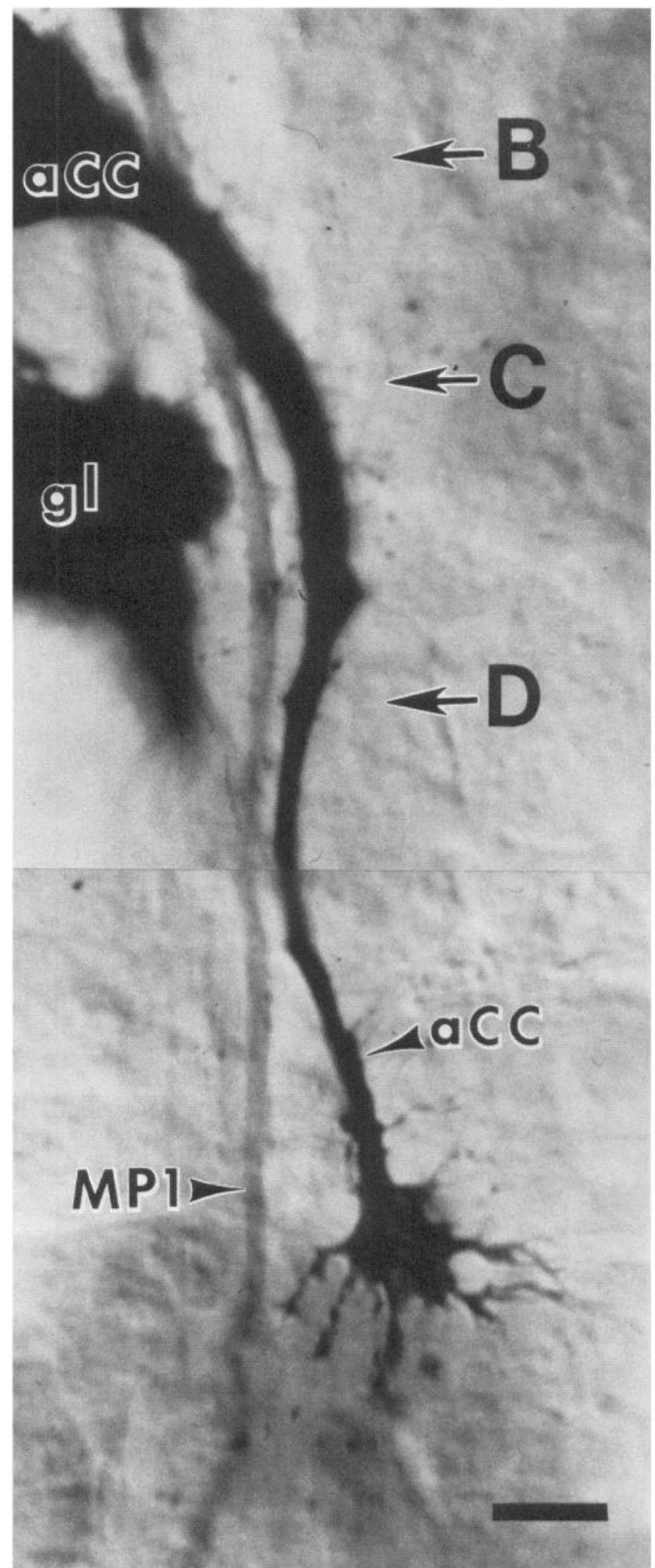

Figure 1. Morphology of the aCC growth cone and axon at $35 \%$ of development, shown in this whole-mount embryo in which the $a C C$ neuron [as well as the $M P 1$ neuron and a glial cell $(g l)$ ] had been filled with HRP. The aCC growth cone is about to turn laterally along the intersegmental nerve at the segment border. This embryo had been prepared for electron microscopy. The arrows and letters $B-D$ indicate the levels of the electron-microscopic sections shown in $B-D$ in Figure 2. Scale bar, $10 \mu \mathrm{m}$. 


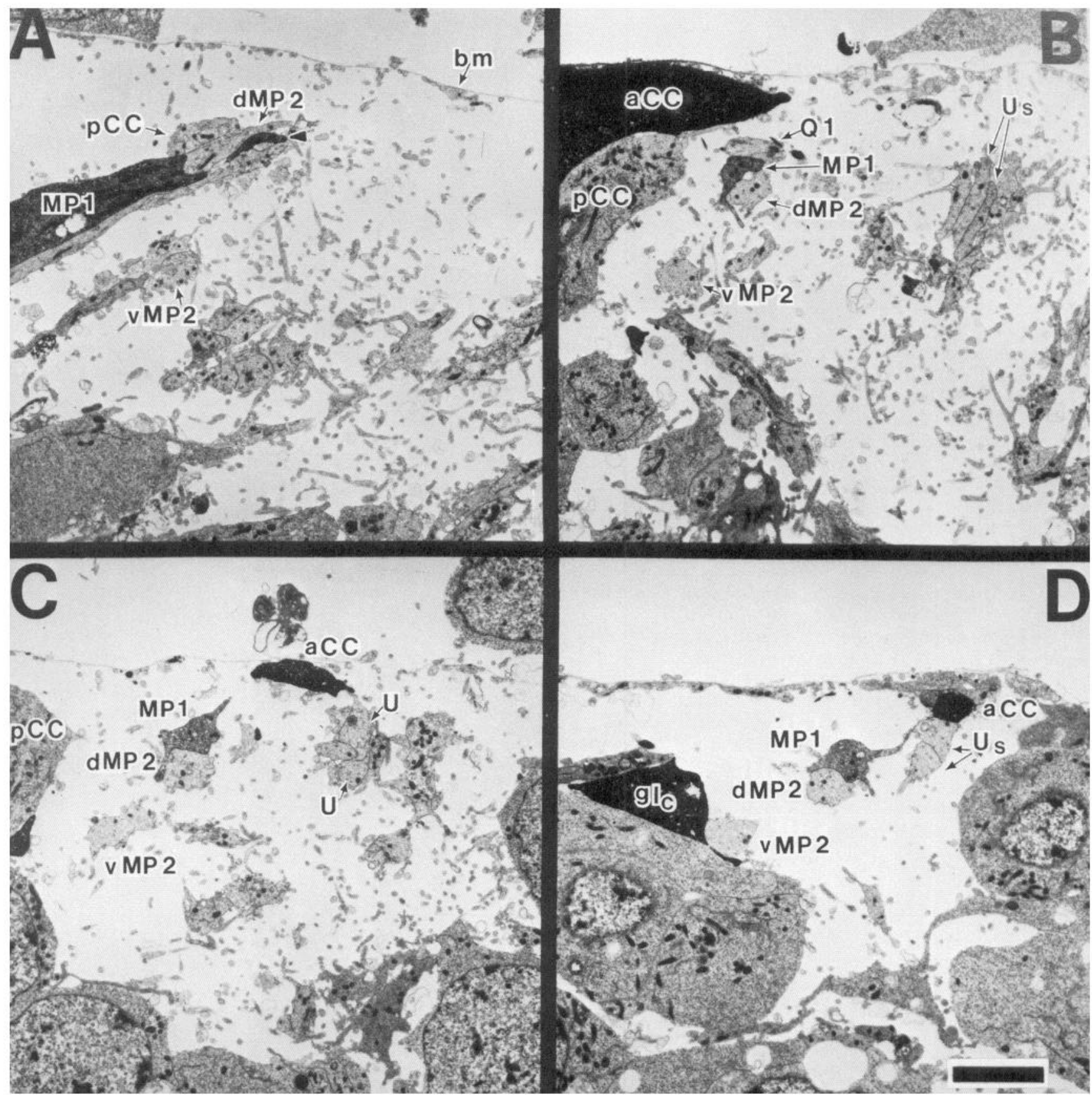

Figure 2. Selective affinity of the pCC growth cone for the MP1/dMP2 axons, and of the aCC growth cone for the U axons, as revealed by HRP injections and serial section electron microscopy. The $M P 1$ and $a C C$ neurons, and an identified glial cell $\left(g l_{c}\right)$, were filled with HRP, as shown in Figure 1. A, Section at the level of the MP1 cell body shows the axon of the filled MP1 extending dorsolaterally from its soma to join the MP1/ $d M P 2$ fascicle, which, at this level, also contains the $p C C$ axon. The $v M P 2$ fascicle is just ventral and medial to the MP1/dMP2 fascicle and, at this stage and level, contains the vMP2 from the next posterior segment (labeled) and the vMP2 from the same segment (unlabeled) (not shown). $\mathrm{bm}$, Basement membrane. $B$, Section through the $a C C$ cell body shows its axon extending laterally just under the basement membrane over the $M P 1 / d M P 2$ fascicle and toward the $\mathrm{U}$ fascicle $(U s)$. The $p C C$ axon extends around the aCC to join the MP1/dMP2 fascicle, as shown in $A$. The axon of the $Q 1$ neuron contacts MP1 as it crosses medially to form one of the first fascicles in the posterior commissure. The $v M P 2$ fascicle contains only the vMP2 axon from the next posterior segment at this level. $C$, At the level of the $p C C$ cell body, the $a C C$ axon is still extending along the dorsal basement membrane toward the $U$ fascicle. The ventromedial $v M P 2$ fascicle contains only the vMP2 axon. The centrally located $M P 1 /$ $d M P 2$ fascicle contains only the filled axon of MP1 and the unfilled axon of dMP2. Note the densely filled profile of the glial cell just ventral to the pCC soma. $D$, Section through the longitudinal connective shows the 3 longitudinal bundles, the $v M P 2, M P 1 / d M P 2$, and $\mathrm{U}$ fascicles $(U s)$. The $a C C$ fasciculates with the two $U(U s)$ axons. The vMP2 axon is in close apposition to the filled profile of the glial cell $\left(g l_{c}\right)$. Scale bar, $2 \mu \mathrm{m}$.

forming a flattened sheet just under the dorsal basement membrane. These aCC filopodia are in a position to contact the nearby axons of the MP1, dMP2, and pCC neurons. At 33\%, the lamellopodia and many of the filopodia shift laterally. At this stage, the U growth cones have emerged at the dorsal basement membrane just lateral to the MP1/dMP2 fascicle, within $10 \mu \mathrm{m}$ and thus within filopodial grasp of the aCC neuron.

As the aCC growth cone begins to extend along the $\mathrm{U}$ axons, 




Figure 3. The growth cone and filopodia of the aCC neuron. Photographs of the aCC neuron in whole-mount embryos in immunostained preparations using anti-LY antibody after intracellular injection of LY. Left, aCC neuron in a 34\% embryo, showing numerous long filopodia extending from the posterior-pointing growth cone; shorter filopodia extend from the cell body. Right, aCC neuron in a $35 \%$ embryo. By this stage, the aCC growth cone has extended posteriorly to the segment border and most of the filopodia emerge from the growth cone. Scale bar, $10 \mu \mathrm{m}$.

it leaves behind an axon. During axonal outgrowth, the site of filopodial extension shifts from the cell body to the growth cone. At $34 \%$, the aCC growth cone points posteriorly. Tufts of long filopodia emerge from the growth cone and are primarily directed posteriorly. At $35 \%$, an axon extends posteriorly to the intersegmental border. The majority of the longer filopodia radiate from the growth cone; shorter, lateral filopodia continue to radiate from the axon and cell body.

We observed the extension and contacts of the aCC filopodia at the ultrastructural level by fixing the embryo, injecting HRP into the aCC cell body, fixing the embryo again, and processing the embryo first for HRP immunocytochemistry and then for electron microscopy. An example of such an aCC neuron, filled with HRP at $32 \%$, is shown in Figure 5 . The two $U$ growth cones have just arrived at the dorsal basement membrane (Fig. $5 B)$. Many filopodia radiate anteriorly from the aCC cell body (arrowheads in Fig. 5A), as well as laterally from its side (arrowheads in Fig. $5 B$ ). The aCC filopodia project as a relatively planar, flattened sheet just under the dorsal basement membrane. In so doing, they contact the 2 bundles of axons near the basement membrane: the MP1/dMP2 fascicle and the U fascicle.

\section{Cell ablation experiments}

The results of the light- and electron-microscopic studies demonstrate that the aCC growth cone has a high affinity for the U1 and $\mathrm{U} 2$ axons. The aCC growth cone appears to specifically use the surfaces of the $U$ axons as a substrate upon which to extend posteriorly. To test the hypothesis that the $\mathrm{U}$ axons play an active role in guiding the aCC growth cone, we ablated the cell bodies of the $U$ neurons with a laser microbeam before they had extended growth cones. The embryos were allowed to develop in a culture system, and then we assayed the aCC neuron using dye injection and immunocytochemical methods.

The development of the aCC, pCC, and neighboring neurons is relatively normal in the culture system; the fasciculation pat- 

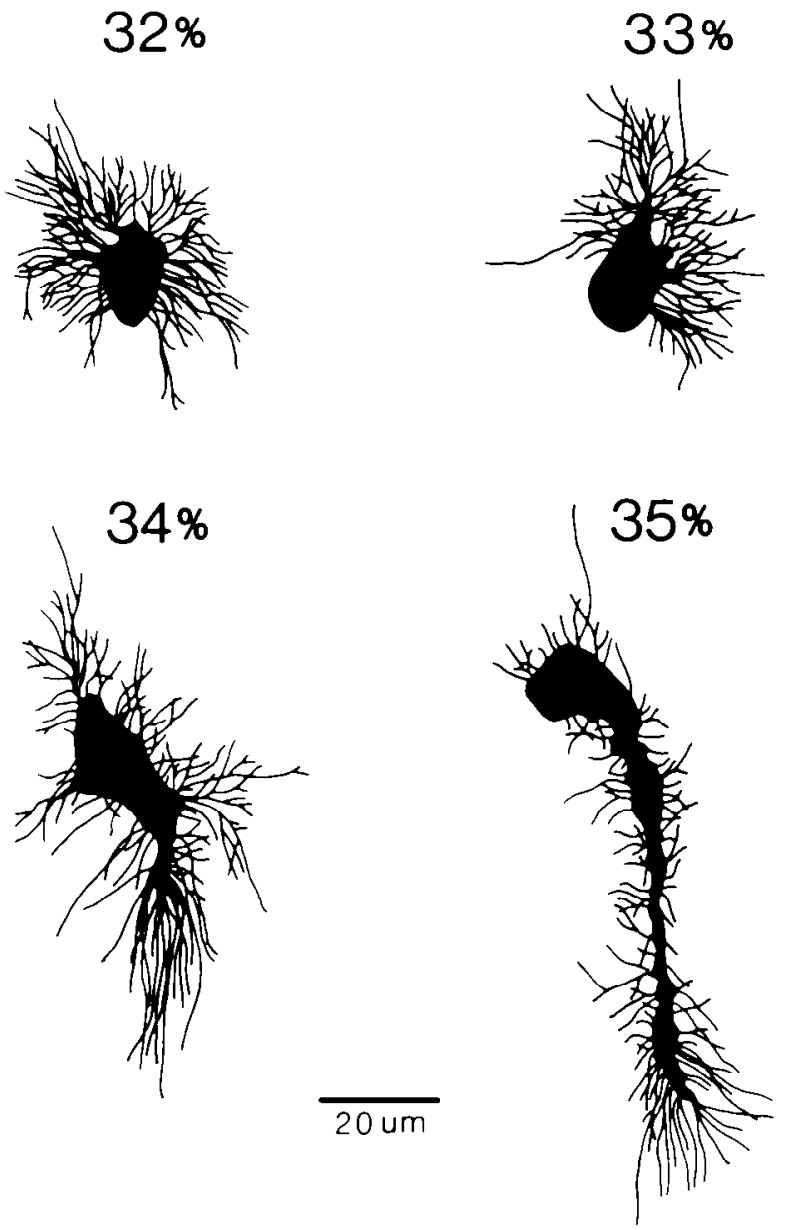

Figure 4. Filopodia of the aCC growth cone. We observed the growth cone and filopodia of the aCC neuron by fixing the embryo, injecting LY into the cell body, fixing the embryo again, incubating the embryo in the anti-LY antibody, and processing the embryo with HRP immunocytochemistry. The shape of the aCC growth cone and the extent of its filopodia change dramatically between 32 and $35 \%$ of development. At $32 \%$, numerous filopodia radiate in a planar halo from the aCC cell body. These filopodia are in a position to contact the nearby axons of the MP1, dMP2, and pCC neurons. At 33\%, the lamellopodia and many of the filopodia are directed laterally. At this stage, the U growth cones have emerged just lateral to the MPl/dMP2 fascicle and thus are within filopodial grasp of the aCC neuron. As the aCC extends an axon, the site of filopodial extension shifts from the cell body to the growth cone. At $34 \%$, the aCC growth cone points posteriorly. Tufts of long filopodia emerge from the growth cone and are directed posteriorly. Many filopodia have retracted from the cell body by this stage. At $35 \%$. an axon extends posteriorly to the intersegmental border. The majority of the longer filopodia radiate from the growth cone; shorter, lateral filopodia continue to radiate from the axon and cell body.

terns and relative timing of neuronal events parallel those in ovo. The absolute rate of development, however, is slower in cultured than in uncultured embryos; $36-48 \mathrm{hr}$ of development in embryo culture is equivalent to about $24 \mathrm{hr}$ of development in ovo. In some cultured embryos, the aCC cell body was displaced laterally from its normal position. In these embryos, axonogenesis proceeded normally.

The $U$ neurons were ablated by focusing a laser microbeam on their somata. Manipulations were performed on only 1 side of the embryo; thus the contralateral hemisegment served as an internal control. Embryos were cultured for 48-60 hr. Upon removal from culture, the embryo was fixed and either (1) it was stained with the I-5 MAb or (2) the aCC neurons were filled bilaterally with LY and the embryo was processed with antiLY and HRP immunocytochemistry. In all I-5 MAb-stained embryos, and in some dye-filled embryos, the morphology of the $p C C$, which served as a control for nonspecific effects of the ablations, was noted.

Experiments were performed at $30 \%$ of development, before the $\mathrm{U}$ neurons had extended growth cones and before the migrating aCC and pCC neurons had reached their final cell body position (Fig. $6 A$ ). This development stage was chosen to avoid having the laser microbeam pass through the growth cone of the aCC en route to the ventrally located $U$ somata. In sham ablations, the laser microbeam was directed at a pair of cells that lie in the same ventral plane as the Us but that are located medial to the $U$ somata (Fig. $6 B$ ). These cells were chosen because at $31 \%$, during the time that the sham ablations were performed, they lie at the same distance from the aCC cell body as do the $U$ neurons at $30 \%$, when the experimental ablations are performed. Thus, the aCC neurons in both sham and experimental cases were exposed to the same peripheral effects of the laser.

The morphologies of experimental and sham-operated control aCC neurons are shown in Figure 7. The 4 examples in Figure 7 are typical of experimental $(A-C)$ and sham $(D)$ results. In a total of 19 embryos in which the $U$ neurons were ablated, the aCC neither extended an axon posteriorly, as it would normally. nor fasciculated with an anomalous axon bundle. Instead, the growth cone usually continued to point anteriorly (Fig. 7 , $A, B$ ) or, occasionally, laterally and posteriorly (Fig. $7 C$ ), in most cases without elongating an axon.

The experimental aCC neuron often resembled that of an unmanipulated, $31-32 \%$ embryo. In normal embryos at $32 \%$ of development, the aCC process measures $10-15 \mu \mathrm{m}$ from the cell body to the tip of the growth cone. In 15 of 19 experimental embryos, the length of the process fell within this range. In 3 embryos, the process measured up to $25 \mu \mathrm{m}$. In one anomalous case, the aCC extended a thin, $60 \mu \mathrm{m}$ process anteriorly along the basement membrane. The axon did not appear to fasciculate with any other axons and wandered lateral to the MPl/dMP2 fascicle.

The control aCC neuron always developed normally. Five of the control axons had the morphology of a normal $34 \%$ aCC, while the remaining 14 had morphologies of normal $35-37 \%$ aCC neurons. Thus, the difference in morphology of experimental and control aCC neurons was over $3 \%$ (and often as much as $5 \%$ ) of development, while normal left-right variation is never more than $0.5 \%$ during the period between 32 and $37 \%$. Sham-operated controls (Fig. $7 D, n=6$ ) gave axon growth equivalent to that on the control side, with no greater than a $0.5 \%$ difference in morphologies. All pCC neurons developed normally.

Another example of an experimental result is given in Figure 8. On the experimental side (Fig. 8.A), the aCC growth cone extended $20 \mu \mathrm{m}$ anteriorly. On the control side (Fig. $8 B$ ), the aCC growth cone first extended posteriorly along the $U$ axons, then turned laterally along the intersegmental nerve; the morphology of the aCC neuron was equivalent to that of a normal $36 \%$ aCC.

\section{Discussion}

Affinities of the aC' and $p C^{\circ} C$ growth cones for specific axonal surfaces

In the previous paper, we described the specific affinity of the pCC growth cone for the MP1 and dMP2 axons. Here we have shown a similarly high degree of specificity of the aCC growth cone for the $U 1$ and $U 2$ axons (Fig. 9). Taken together, these 2 sets of descriptive and experimental results provide strong support for the labeled-pathways hypothesis (Bastiani et al., 1984; 


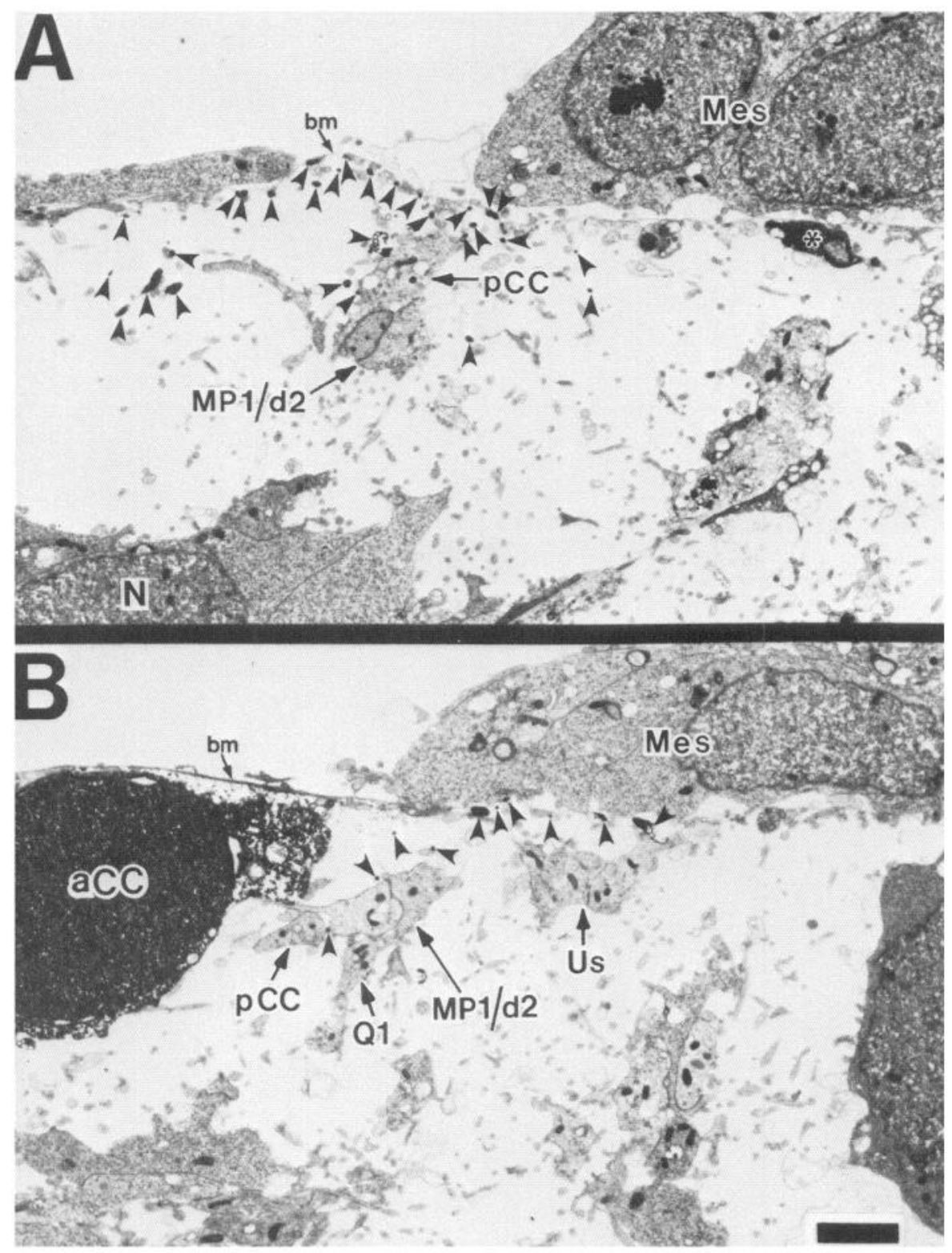

Figure 5. Filopodial contacts of the aCC neuron before it turns onto the $\mathrm{U}$ fascicle. Electron micrographs of a $32 \%$ embryo in which the aCC neuron had been filled with HRP. A, Section anterior to aCC cell body, showing relatively planar radiation of filopodia dorsally near the basement membrane $(\mathrm{bm})$. Many filopodia are in contact with the $M P 1, \mathrm{dMP} 2(d 2)$, and $p C C$ axons. $N$, Neuronal cell body; Mes, mesodermal cell. $B$, Section at level of the $a C C$ cell body, showing the lateral filopodia dorsal near the basement membrane $(\mathrm{bm})$ contacting both the MP1/dMP2 (d2) fascicle and the U growth cones $(U s)$ that have just arrived at the $\mathrm{bm}$. Note relatively dorsal distribution of aCC filopodia. Mes, Mesodermal cell. Scale bar, 2 $\mu \mathrm{m}$.

Goodman et al., 1982; Raper et al., 1983a-c, 1984). The aCC and pCC growth cones both appear to demonstrate absolute preferences, rather than hierarchical ones, for specific axonal pathways. These experiments support the notion that the surfaces of the $2 \mathrm{U}$ axons and of the MP1/dMP2 axons have special distinguishing labels that guide the aCC and pCC growth cones, respectively, onto and along their surfaces.

Although the experiments described here indicate that the aCC growth cone requires specific interactions with the $U$ neurons, they do not allow us to distinguish between a specific requirement for axon initiation only and a maintained requirement for continued axon extension. However, experiment 3 of the previous paper tests these alternatives for the pCC growth cone and indicates that specific axons are required not only for axon initiation but for continued axon extension as well.

In the previous paper, experimental results with the pCC growth cone were used to argue against (1) the simple location of the axons, (2) simple timing of axon outgrowth, and/or (3) simple quantitative differences in the expression of a common surface label being the major determinant in growth cone guidance. The experimental results with the $\mathrm{aCC}$ growth cone pre- sented here provide further evidence for these arguments.

(1) If simple location were the determinant, then we might expect the aCC growth cone to turn posteriorly along the MP1/ dMP2 fascicle, which is only $1-2 \mu \mathrm{m}$ away from where the $U$ fascicle develops. The aCC growth cone has easier access to the surface of the MP1/dMP2 fascicle, which also extends posteriorly in the same direction as the $U$ fascicle. Normally, the aCC growth cone must extend across the MP1/dMP2 fascicle to reach the $U$ fascicle, which is several microns on the other side. (2) The normal 10-15 hr delay in the extension of the aCC growth cone (and the precise correlation between the timing of its extension and the arrival of the $U$ axons) argues against subtle timing mechanisms. If the aCC simply waited for some arbitrary period before being ready for axonogenesis, then, in the absence of the Us, other axons should serve as a suitable substrate, but they do not. (3) Finally, if a simple quantitative difference in the expression of a common surface label was involved, we might expect to see a hierarchy of pathway choice in the absence of the Us. However, the aCC growth cone, rather than picking another fascicle, does not show a high affinity for any other axon (Fig. 9). 
A

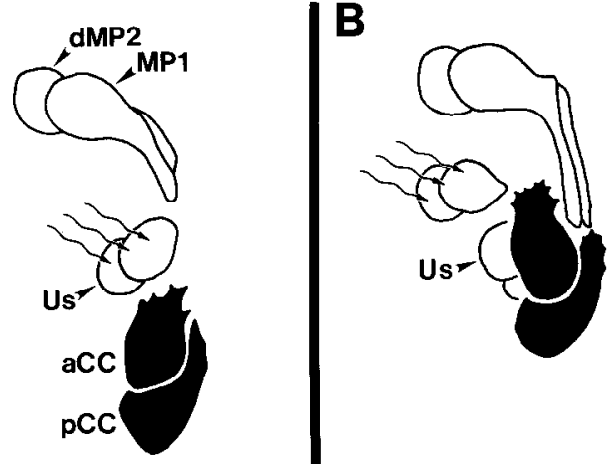

Figure 6. Diagram showing relative positions of cell bodies during experimental $(A)$ and sham $(B)$ ablations with a laser microbeam. Camera lucida drawings of embryos stained with the I- $5 \mathrm{MAb}$ at the time of experimental and sham ablations. $A$, Laser ablation of the $\mathrm{U}$ somata $(U s)$. Experiments were performed at $30 \%$, before the $a C C$ had reached its final position dorsal to the Us and before the Us had extended growth cones. $B$. Sham-operated controls. A pair of cells situated anteromedial to the $\mathrm{U}$ somata $(U s)$ was killed at $31 \%$ after the aCC had reached its final position. See text for details.

Implicit in our application of the labeled-pathways hypothesis to the behavior of the aCC and pCC growth cones is the notion that these growth cones actively sample the axons in their environment through their profuse radiation of filopodia. In tissue culture, filopodial adhesion appears to mediate growth cone guidance (Bray, 1982; Letourneau, 1982). Our observations of the filopodia of the aCC growth cone support the hypothesis that the aCC filopodia specifically recognize the $U$ axons. Prior to the arrival of the $\mathrm{U}$ growth cones to within $10 \mu \mathrm{m}$ and, thus, within filopodial grasp of the aCC neuron, the aCC radiates filopodia in a planar halo that contacts the MP1/dMP2 fascicle and quite likely other axons as well.

As the U growth cones reach the dorsal basement membrane just lateral to the MP1/dMP2 fascicle and extend posteriorly, the morphology of the aCC neuron changes dramatically. The aCC filopodia shift laterally towards the $U$ axons; the aCC filopodia then appear to guide the aCC growth cone as it turns laterally and then posteriorly along the $U$ axons. Although the aCC filopodia have access to many axons in the developing longitudinal connective, the aCC growth cone selects only the $\mathrm{U}$ axons upon which to extend, and in their absence remains relatively stationary.
A

\section{EXP.}

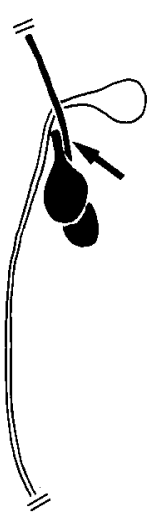

\section{CONTROL}

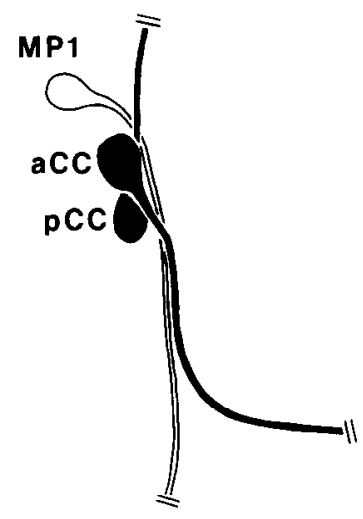

\section{EXP.}

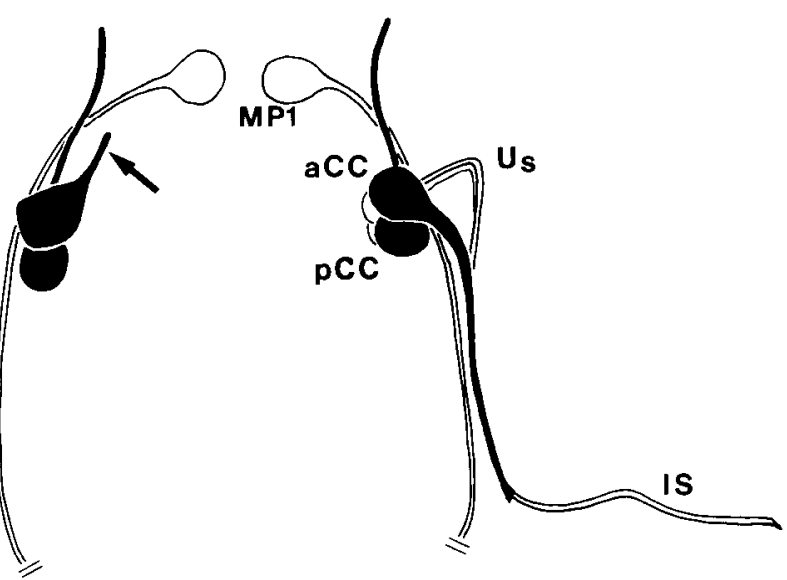

EXP.

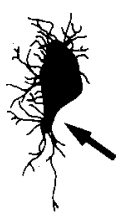

\section{CONTROL}

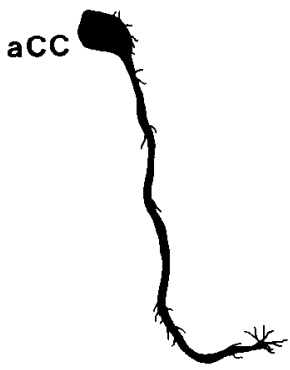

SHAM

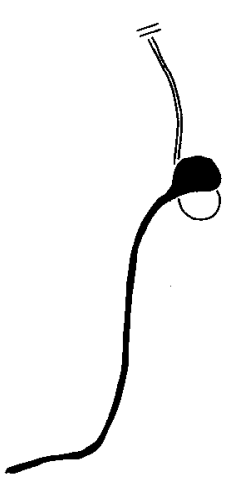

CONTROL

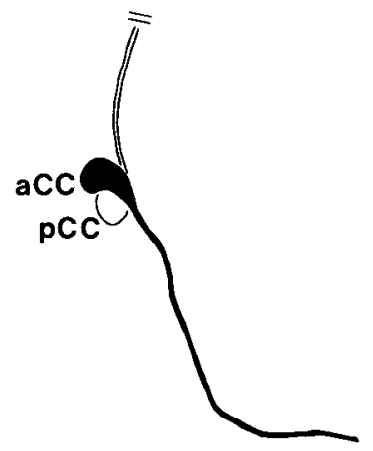




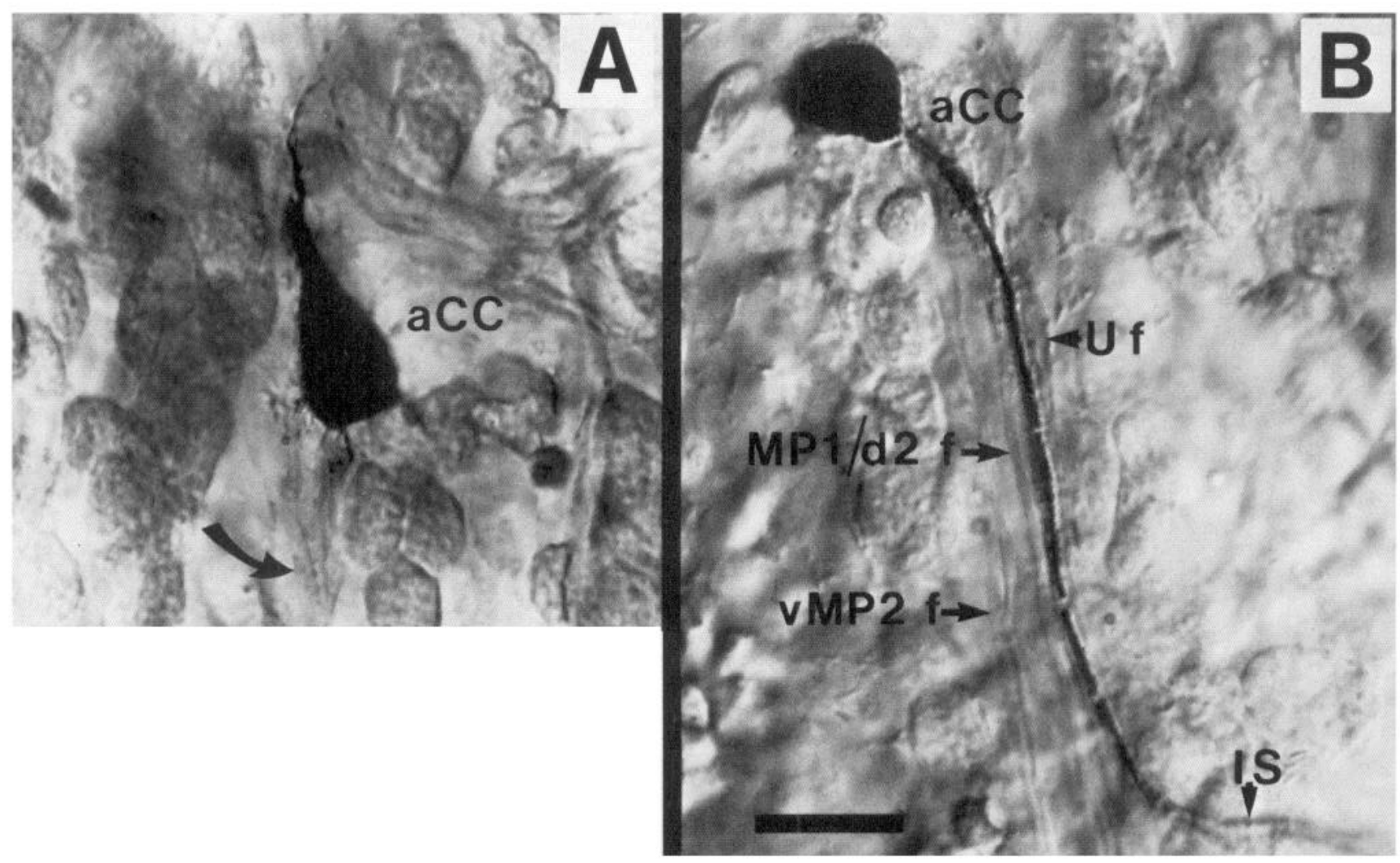

Figure 8. Photomicrograph of an experimental embryo in which the $\mathrm{U}$ cell bodies were ablated in the left hemisegment $(A)$, the embryo was cultured for $50 \mathrm{hr}$, and the aCC neurons were filled bilaterally with LY and stained with anti-LY antibody. $A$, The $a C C$ neuron on the experimental side extended a growth cone for a short distance anteriorly but did not fasciculate with any other axons, including those fascicles remaining in the longitudinal connective (arrow). B, The aCC neuron on the control side extended an axon posteriorly in the $U$ fascicle $(U f)$ and laterally along the intersegmental nerve $(I S)$ as in normal, uncultured embryos. MP1/d2 $f$ marks the MP1/dMP2 fascicle; $v M P 2 f$ marks the vMP2 fascicle. Scale bar, $20 \mu \mathrm{m}$.

\section{Labeled-pathways hypothesis: cell recognition by neuronal growth cones}

In this and the previous paper, we have described 2 examples of growth cone specificity that, together with the studies on the $\mathrm{G}$ growth cone (Bastiani et al., 1984; Raper et al., 1983a-c, 1984), support the hypothesis that recognition molecules are differentially expressed on the surfaces of different embryonic axon fascicles, or subsets of axons within them. These postulated recognition molecules are likely to guide neuronal growth cones as they make specific choices to follow particular pathways and tracts to their appropriate targets.

How many recognition molecules exist remains an open question. Certainly, every neuron does not have its own unique chemical label; the model predicts unique pathway labels, not unique neuronal labels. Passive and spatiotemporal constraints no doubt help reduce the number of molecules needed to channel growth cones into certain pathways. But, in addition, active mechanisms are required; growth cones make specific and divergent choices that implicate different molecules in pathway and target recognition.

It is important to point out that cell recognition by neuronal growth cones is only one of many mechanisms that interact over space and time to generate a precisely wired nervous system. Neuronal specificity begins to unfold as the mechanisms of growth-cone guidance give rise to a basic scaffold of axonal projections that places each neuron's processes in a local neighborhood, properly juxtaposed to its appropriate targets. These events are followed by the activity-dependent pruning and refining of these stereotyped projections and connections; such

Figure 7. The morphology of the aCC neuron in experimental embryos. Camera lucida drawings of antibody-stained embryos in which the U cells $(A-C)$ or sham-operated target cells $(D)$ were ablated with a laser microbeam. $A$, I-5 MAb-stained embryo in which the U cell bodies were ablated at $30 \%$ and the embryo cultured for $56 \mathrm{hr}$. The control aCC extended posteriorly upon the U fascicle as normal. The experimental (exp) aCC growth cone (arrow), in the absence of the Us, did not fasciculate with any other axons but instead remained pointed anteriorly. The $p C C$ in both control and experimental sides developed normally. B. Another example of an I-5 MAb-stained experimental embryo; here the embryo was cultured for $48 \mathrm{hr}$. The control $\mathrm{U}$ axons $(U s)$ have extended posteriorly and then laterally along the intersegmental nerve (IS). The $a C C$ is equivalent to that of a normal $35 \%$ embryo and has followed the Us posteriorly. On the experimental (exp.) side, in the absence of the U axons, the aCC growth cone (arrow) points anteromedially without fasciculating with other axons. $C$, Experimental embryo in which, following ablation of the $\mathrm{U}$ cell bodies, the embryo was cultured for $54 \mathrm{hr}$ and the aCC neurons were filled bilaterally with LY and processed with anti-LY antibody. The control $a C C$ axon extends posteriorly and then laterally out the intersegmental nerve. The experimental (exp.) aCC has a small, laterally and posteriorly pointed process (arrow) radiating filopodia in an apparently undirected fashion. $D$, Sham-operated control in which the $a C C$ and $p C C$ neurons were filled bilaterally with LY and stained with the anti-LY antibody; both sham-operated and control $a C C$ and $p C C$ neurons developed normally. Scale bar, $60 \mu \mathrm{m}(A, D) ; 50 \mu \mathrm{m}(B, C)$. 

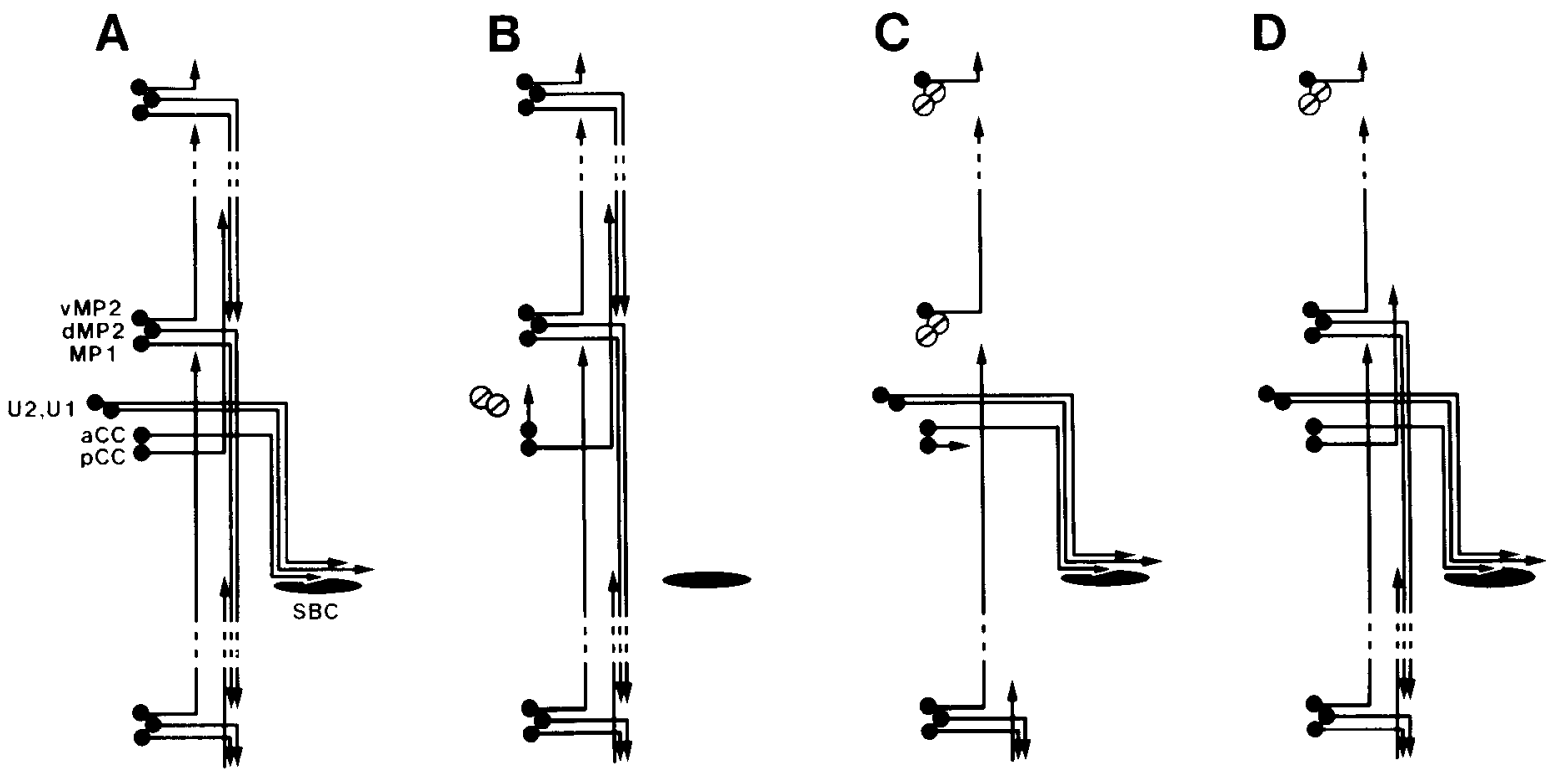

Figure 9. Schematic diagram summarizing the axons in the first 3 longitudinal axon fascicles and intersegmental nerve in the grasshopper embryo $(A)$, and the results of 3 different cell ablation experiments described here $(B)$ and in the previous paper $(C, D)$. $B-D$, In vitro ablation experiments in which the $\mathrm{U}$ neurons $(B), \mathrm{MP} 1$ and dMP2 neurons in the test and next anterior segment $(C)$, and MP1 and dMP2 neurons in the next anterior segment only $(D)$ had been ablated. The results demonstrate the selective affinities of the aCC growth cone for the $\mathrm{U}$ fascicles, and of the pCC growth cone for the MP1/dMP2 fascicle. For further discussion, see text.

synaptic rearrangement gives rise to the precise patterns of connection within each local neighborhood.

\section{Generality of the model}

Evolutionary arguments and empirical observations suggest that similar mechanisms of growth-cone guidance play a significant role during the development of all organisms - from nematodes, to insects, to lower vertebrates, to man. In both invertebrates and vertebrates, experimental results implicate specific guidance cues and active choices made by growth cones as they follow stereotyped pathways to their targets (e.g., Kuwada, 1986; LanceJones and Landmesser, $1981 \mathrm{la}, \mathrm{b}$; Tosney and Landmesser, 1984, 1985a, b).

Insect nervous systems contain about $10^{5}$ neurons and the human nervous system about $10^{12}$ neurons, with other vertebrates somewhere between these two. Whereas an insect has one or only a few identified neurons subserving a particular function, a vertebrate has from thousands to hundreds of thousands of similar neurons subserving this function. The labeled-pathways model applies to axonal pathways and tracts in vertebrates just as it does in invertebrates, except that, in vertebrates, these pathways are likely to contain one to several orders of magnitude more axons than in invertebrates. For example, we would predict that growth cones could distinguish between specific longitudinal tracts in the spinal cord, just as they can distinguish between the MP1/dMP2 and U fascicles.

Constantine-Paton and Capranica $(1975,1976)$ showed that transplanted optic axons always follow a particular axon tract in the spinal cord, regardless of where they enter the cord. Similarly, Katz and Lasek $(1979,1981)$ showed that transplanted Mauthner axons and optic axons take different, yet stereotyped, tracts within the spinal cord. Recent experiments have demonstrated selective fasciculation by the growth cones of identified interneurons in the developing spinal cord of the fish embryo (Kuwada, 1986). Moreover, in tissue culture, growth cones of vertebrate neurons can distinguish between particular axonal surfaces in a cell-specific manner (Bray et al., 1980; Raper et al., 1985).
Just as the longitudinal axon tracts in the grasshopper often contain further specificities within them, so the tracts in vertebrates may contain further specificities as well. For example, Bonhoeffer and Huf (1985) have recently demonstrated the sclective affinity of growth cones from the temporal retina for axons from the temporal retina, rather than from the nasal retina, suggesting that the optic tract may contain graded nasaltemporal specificities.

At this juncture it is difficult to say with certainty just how many recognition molecules exist and how similar their function is in developing invertebrate and vertebrate nervous systems. Sufficient biochemical and functional data are not yet at hand in any organism. Over the next few years, however, it may be possible to better understand the mechanisms of neuronal recognition by combining a cellular analysis of grasshopper, as described here, with a molecular genetic analysis of Drosophila (e.g., Goodman et al., 1984; Thomas et al., 1984).

\section{References}

Bastiani, M. J., and C. S. Goodman (1986) Guidance of neuronal growth cones in the grasshopper embryo. III. Recognition of specific glial pathways. J. Neurosci. 6: 3542-3551.

Bastiani, M. J., S. du Lac, and C. S. Goodman (1986) Guidance of neuronal growth cones in the grasshopper embryo. I. Recognition of a specific axonal pathway by the pCC neuron. J. Neurosci. 6: 35183531 .

Bastiani, M. J., J. A. Raper, and C. S. Goodman (1984) Pathfinding by neuronal growth cones in grasshopper embryos. III. Selective affinity of the $G$ growth cone for the $P$ cells within the $A / P$ fascicle. J. Neurosci. 4: 2311-2328.

Bastiani, M. J., C. Q. Doe, S. L. Helfand, and C. S. Goodman (1985) Neuronal specificity and growth cone guidance in grasshopper and Drosophila embryos. Trends Neurosci. 8: 257-266.

Bonhoeffer, F., and J. Huf (1985) Position-dependent properties of retinal axons and their growth cones. Nature 315: 409-410.

Bray, D. (1982) Filopodial contraction and growth cone guidance. In Cell Behavior, R. Bellairs, A. Curtis, and G. Dunn, eds., pp. 299317, Cambridge U. P., Cambridge, UK.

Bray, D., P. Wood, and R. P. Bunge (1980) Selective fasciculation of nerve fibres in culture. Exp. Cell Res. 130: 241-250. 
The Journal of Neuroscience

Recognition of a Specific Axonal Pathway by the aCC Neuron

3541

Constantine-Paton, M., and R. P. Capranica (1975) Central projection of optic tract from translocated eyes in the leopard frog (Ran pipiens). Science 189: 480-482.

Constantine-Paton, M., and R. P. Capranica (1976) Axonal guidance of developing optic nerves in the frog. I. Anatomy of the projection from transplanted eye primordial. J. Comp. Neural. 170: 17-32.

Doe, C. Q., and C. S. Goodman (1985) Early events in insect nurogenesis: II. The role of cell interactions and cell lineage in the determination of neuronal precursor cells. Lev. Biol. 111: 206-219.

Goodman, C. S., J. A. Raper, K. K. Ho, and S. Chang (1982) Pathfinding of neuronal growth cones in grasshopper embryos. In Bevelopmental Order: Its Origin and Regulation, S. Subtelny and P. B. Green, eds., pp. 275-316, Liss, New York.

Goodman, C. S., M. J. Bastiani, C. Q. Doe, S. du Lac, S. L. Helfand, J. Y. Kuwada, and J. B. Thomas (1984) Cell recognition during neuronal development. Science 225: 1271-1279.

Katy, M. J., and R. J. Lase (1979) Substrate pathways which guide growing axons in Xenopus embryos. J. Comp. Neural. 183: 817-832.

Katy, M. J., and R. J. Lase (1981) Substrate pathways demonstrated by transplanted Mauthner axons. J. Comp. Neural. 195: 627-641.

Kuwada, J. Y. (1986) Cell recognition by neuronal growth cones in a simple vertebrate embryo. Science 233: 740-746.

Lance-Jones, C., and L. Landmesser (1981a) Pathway selection by chick lumbrosacral motoneuron during normal development. Proc. R. Soc. Lond. [Biol.] 214: 1-18.

Lance-Jones, C., and L. Landmesser (1981b) Pathway selection by embryonic chick motoneuron in an experimentally altered environmont. Proc. R. Soc. Lond. [Biol.] 214: 19-52.

Letourneau, P. C. (1982) Nerve fiber growth and its regulation by extrinsic factors. In Neuronal Development, N. C. Spitzer, ed., p. 213254, Plenum, New York.
Raper, J. A., M. J. Bastiani, and C. S. Goodman (1983a) Pathfinding by neuronal growth cones in grasshopper embryos. I. Divergent choices made by the growth cones of sibling neurons. J. Neurosci. 3: 20-30.

Raper, J. A., M. J. Bastiani, and C. S. Goodman (1983b) Pathfinding by neuronal growth cones in grasshopper embryos: II. Selective asciculation onto specific axonal pathways. J. Neurosci. 3: 31-41.

Raper, J. A., M. J. Bastiani, and C. S. Goodman (1983c) Guidance of neuronal growth cones: Selective fasciculation in the grasshopper embryo. Cold Spring Harbor Symp. Quant. Biol. 48: 587-598.

Raper, J. A., M. J. Bastiani, and C. S. Goodman (1984) Pathfinding by neuronal growth cones in grasshopper embryos. IV. The effects of ablating the $A$ and $P$ axons upon the behavior of the $G$ growth cone. J. Neurosci. 4: 2329-2345.

Raper, J. A., J. P. Kapfhammer, and E. B. Grunewald (1985) Contact mediated avoidance between specific pairs of growth cones and newrites in culture. Soc. Neurosci. Abstr. 11: 176.

Taghert, P., M. J. Bastiani, R. K. Ho, and C. S. Goodman (1982) Guidance of pioneer growth cones: Filopodial contacts and coupling revealed with an antibody to Lucifer Yellow. Div. Biol. 94: 391-399.

Thomas, J. B., M. J. Bastiani, C. M. Bate, and C. S. Goodman (1984) From grasshopper to Drosophila: A common plan for neuronal development. Nature 310: 203-207.

Tosney, K. W., and L. T. Landmesser (1984) Pattern and specificity of axonal outgrowth following varying degrees of chick limb bud ablation. J. Neurosci. 4: 2518-2527.

Tosney, K. W., and L. T. Landmesser (1985a) Specificity of early motoneuron growth cone outgrowth in the chick embryo. J. Neurosci. 5: 2336-2344.

Tosney, K. W., and L. T. Landmesser (1985b) Growth cone morphology and trajectory in the lumbosacral region of the chick embryo. J. Neurosci. 5: 2345-2358. 